\title{
Evaluation of Global Sagittal Balance in Koreans Adults
}

\author{
Yongjae Cho, M.D., Ph.D. \\ Department of Neurosurgery, Ewha Womans University College of Medicine, Seoul, Korea
}

Objective : The global sagittal postural patterns as characterized by Roussouly classification have been previously described in various ethnicities, there were no studies investigated in Koreans. To analyze the distribution of the global sagittal postural patterns in Korean adults using Roussouly classification, the author prospectively studied.

Methods : 252 asymptomatic Korean adults was recruited. Data was obtained by reviewing the films for each subject. Spinopelvic parameters were measured and sagittal postural patterns were then determined according to Roussouly classification. We compared the data across different ethnicities from our study and a previous study to further characterize Korean sagittal postures.

Results : The subject included 151 males and 101 females, with mean age of $33.2 \pm 8.2$ years. The average descriptive results were as below : thoracic kyphosis $28.6 \pm 7.7^{\circ}$, lumbar lordosis $48.3 \pm 10.2^{\circ}$, sacral slope $37.8 \pm 5.8^{\circ}$, pelvic incidence $45.1 \pm 7.5^{\circ}$, pelvic tilt $9.4 \pm 6.7^{\circ}$, spinosacral angle $130.1 \pm 5.4^{\circ}$, and sagittal vertical axis $16.25 \pm 22.5 \mathrm{~mm}$. 125 subjects among $252(49.6 \%)$ belonged to Roussouly type 3 (namely neutral). There were also 58 (23\%), 33 (13.1\%), and 36 (14.3) subjects in type 1, 2, and 4 (namely non-neutral), respectively.

Conclusion : Enrolling 252 asymptomatic Korean adults, this prospective study found that $49.6 \%$ of asymptomatic Korean adults possessed a sagittal posture of Roussouly type 3. All radiologic parameters follows general concept of spinal sagittal balance pattern. Overall, this study might be a basis for further investigation of spinal sagittal balance.

Key Words : Spinal curvatures $\cdot$ Spine $\cdot$ Postural balance.

\section{INTRODUCTION}

The human vertebral column consists of a three-dimensional structure such as sagittal, coronal, and axial plane, and the sagittal plane has various shapes depending on the individual $^{4)}$. The distinctions between normal and pathologic curvatures are less clear in the sagittal plane than the coronal plane ${ }^{1)}$. Various sagittal planes are closely related to back pain and are important factors in the development of various spinal diseases. The spinal sagittal plane was studied relatively late compared to the coronal plane. Global spinal balance refers to the overall alignment of the spine-generally using center of $\mathrm{C} 7$ vertebral body as a reference point-with respect to another reference point on the sacrum or pelvis ${ }^{9)}$. Clinically, global spinal balance is an important aspect of the evaluation of patients with spinal pathology and of surgical planning, to minimize complications such as adjacent segment disease, sagittal imbalance, pseudarthrosis, and progressive deformity ${ }^{9)}$.

In 2004, Roussouly et al. introduced a four type classifica-

- Received : December 7, 2016 •Revised : March 28, 2017 •Accepted : April 25, 2017

- Address for reprints : Yongjae Cho, M.D., Ph.D.

Department of Neurosurgery, Ewha Womans University College of Medicine, 1071 Anyangcheon-ro, Yangcheon-gu, Seoul 07985, Korea Tel : +82-2-2650-2658, Fax : +82-2-2655-2652, E-mail : yongcho@ewha.ac.kr

This is an Open Access article distributed under the terms of the Creative Commons Attribution Non-Commercial License (http://creativecommons.org/licenses/by-nc/4.0) which permits unrestricted non-commercial use, distribution, and reproduction in any medium, provided the original work is properly cited. 
tion through the prospective radiographic study of 160 asymptomatic Caucasian adults, they claimed that among 4 types, type 3 was considered as balanced neutral position and the other types as non-neutral ${ }^{12)}$ (Fig. 1). The classification was mainly based on lumbar alignments and spinopelvic parameters such as sacral slope (SS), lumbar lordosis (LL), numbers of lumbar vertebrae in lordosis, inflection point (apex of lumbar lordosis) and lumbar tilt (Fig. 2). Among those parameters, location of apex of lumbar lordosis and sacral slope was considered most importantly. Roussouly et al. ${ }^{12)}$ asserted that only $37.5 \%$ of asymptomatic Caucasian adults was type 3. MacThiong et al. ${ }^{9}$ characterized the global sagittal balance in a larger cohort of asymptomatic Caucasian adults and emphasized the importance of the global sagittal balance such as surgical planning, evaluation of spinal pathology, postoperative adjacent segment changes, spinal instability, and spinal pseudoarthopathy.

As interests in sagittal balance is growing recently, many studies has been performed to reveal the global sagittal balance patterns of different ethnic groups ${ }^{5,9,17)}$. In Asian population, Hu et al. ${ }^{5)}$ published that $47.8 \%$ of the asymptomatic Chinese belonged to type 3 and whose average pelvic incidence (PI) value was $46.4 \pm 9.6^{\circ}$. There has been a lack of studies about the global sagittal postural patterns in asymptomatic Korean adults. Though some Korean authors studied each

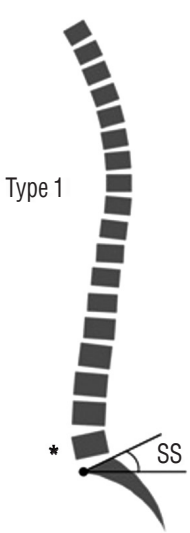

$\mathrm{SS}<35^{\circ}$

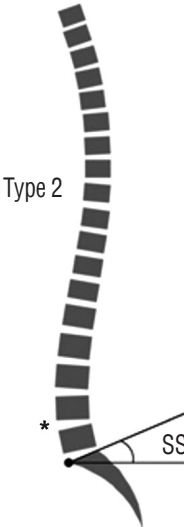

$\mathrm{SS}<35^{\circ}$

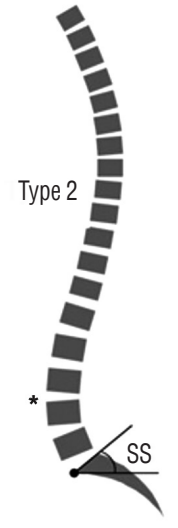

$35^{\circ}<S S<45^{\circ}$

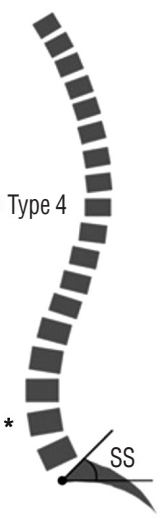

$\mathrm{SS}<45^{\circ}$
Fig. 1. Drawings of Roussouly types. Type $1: \mathrm{SS}<35^{\circ}$, apex of lumbar lordosis (LL) at middle $\mathrm{L} 5$, the spine is hypolordotic and relatively normokyphotic; type $2: \mathrm{SS}<35^{\circ}$, apex of $\mathrm{LL}$ at base $\mathrm{L} 4$, the spine is hypolordotic and hypokyphotic; type $3: 35^{\circ}<\mathrm{SS}<45^{\circ}$, apex of $\mathrm{LL}$ at middle $L 4$, the spine is well balanced; type $4: S S>45^{\circ}$, apex of $L L$ at base $L 3$, the spine is hyperlordotic and hyperkyphotic. *Apex of lumbar lordosis (inflection point). SS : sacral slope. sagittal balance parameter without the analysis of the global sagittal patterns ${ }^{8)}$. Therefore, for the purpose of analyze the global sagittal patterns of asymptomatic Korean adults, we performed this prospective imaging study and compared it with the previous studies of the global sagittal pattern of other ethnic group.

\section{MATERIALS AND METHODS}

\section{Subjects}

A total of 252 adult were enrolled in this study from January to December in 2015. Informed consent was obtained from each one and the Institutional Review Board in Ewha Mokdong Hospital approved the study. Inclusion criteria was normal adults who had visited Ewha Mokdong Hospital in order to the medical check-up in the range of 20 to 50 years old for 1 year from January to December in 2015. All subjects was living in metropolitan urban area. The subjects were mainly comprised of desk workers and housekeepers in occupation style.

252 asymptomatic Korean adults including 151 males and 101 females, with an average age of $33.2 \pm 8.2$ years (ranging from 20 to 48 years old) was prospectively recruited (Table 1). At the time of enrollment, subjects were questioned about their medical history. The subjects who had any of the following conditions were excluded : 1) any history of spinal or or-
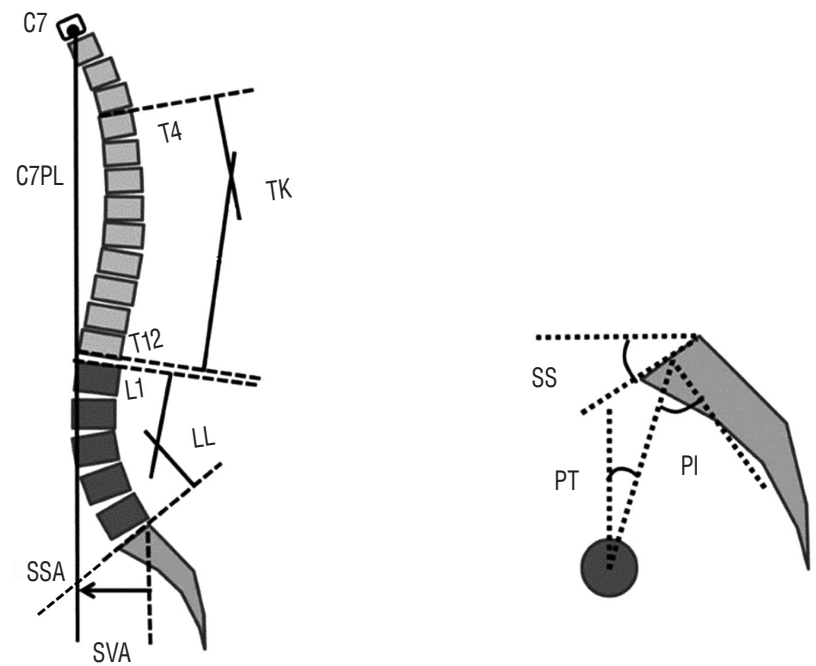

Fig. 2. Measurement of spinopelvic parameters. C7PL : C7 plumb-line, TK : thoracic kyphosis, LL : lumbar lordosis, SSA : spinosacral angle, SVA : sagittal vertical axis, SS : sacral slope, PT : pelvic tilt, PI : pelvic incidence. 
Table 1. Comparison of spinal sagittal parameters between males and females

\begin{tabular}{lcccc}
\hline Variable & All & Male & Female & $p$-value* \\
\hline Age (years) & $33.2 \pm 8.2$ & $35.2 \pm 3.2$ & $32.2 \pm 6.4$ & 0.887 \\
Thoracic kyphosis $\left(^{*}\right)$ & $28.6 \pm 7.7$ & $25.6 \pm 9.3$ & $24.5 \pm 8.5$ & 0.435 \\
\hline Lumbar lordosis $\left(^{\circ}\right)$ & $48.3 \pm 10.2$ & $40.4 \pm 9.7$ & $51.3 \pm 11.2$ & 0.021 \\
Sacral slope $\left(^{\circ}\right)$ & $37.8 \pm 5.8$ & $36.4 \pm 7.2$ & $38.7 \pm 9.2$ & 0.456 \\
Pelvic incidence $\left(^{\circ}\right)$ & $45.1 \pm 7.5$ & $45.1 \pm 9.0$ & $48.5 \pm 9.8$ & 0.059 \\
Pelvic tilt $\left(^{\circ}\right)$ & $9.4 \pm 6.7$ & $8.6 \pm 7.9$ & $10.3 \pm 2.3$ & 0.077 \\
Spinosacral angle $\left(^{\circ}\right)$ & $130.1 \pm 5.4$ & $129.0 \pm 8.7$ & $131.8 \pm 3.4$ & 0.068 \\
Sagittal vertical axis $(\mathrm{mm})$ & $16.25 \pm 22.5$ & $13.93 \pm 36.8$ & $17.79 \pm 22.0$ & 0.551 \\
\hline
\end{tabular}

Values are presented as mean \pm standard deviation. ${ }^{*}$ Comparison in gender, Statistical significance at $p<0.05$, unpaired student t-test

thopedic diseases or trauma, such as back, pelvic, and knee disease; and 2) any abnormal radiologic findings, such as kyphosis, scoliosis, spondylolisthesis, pelvic disease, compressed vertebral fracture beyond the normal range of variation.

\section{Methods}

\section{Imaging methods}

Standing posteroanterior and lateral view is taken with 36-inch-long cassette at Ewha Mokdong Hospital. For subsequent comparison with Caucasian and Chinese adults, this study adopted the radiographic protocol as in Roussouly et al.'s study ${ }^{12)}$. The difference of imaging methods between Roussouly et al.'s study ${ }^{12)}$ and this study was both arm and shoulder position. In this study, fists-on-clavicle position was performed for the avoidance of covering spinal column by arms and shoulders.

The subjects look forward and stands with elbow flexed. The hands are held in a relaxed state and the proximal wrist joint is placed in the center of the clavicle (fists-on-clavicle position). Because the posture of the lower limb affects the balance of the sagittal plane, it is important to maintain the standardized leg posture in the hip joint and knee joint extended state. The distance from the radiographic source to the film was maintained at $1.8 \mathrm{~m}$ and the edges of the films were squared with regard to the horizontal and vertical axes. All radiologic data were archived via Picture Archiving and Communication Systems (PACS; GE Healthcare, Milwaukee, WI, USA). Then the data were retrieved and measured through PACS using the PC-based measuring program.

\section{Radiologic parameters}

The sagittal spinopelvic parameters to measure included : 1) PI, the angle between the line from the center of the femoral head to the midpoint of the sacral endplate and a line perpendicular to the sacral endplate; 2) SS, the angle between the sacral endplate and a horizontal reference line; 3) pelvic tilt (PT), the angle between a line from the center of the femoral head to the midpoint of the sacral endplate and a vertical reference line drawn through the center of the femoral head; 4) spinosacral angle (SSA), the angle subtended by the upper sacral endplate and the line from the center of $\mathrm{C} 7$ vertebral body to the midpoint of upper sacral endplate; 5) LL, the angle subtended by the superior endplates of L1 and S1; 6) thoracic kyphosis (TK), the angle subtended by the superior endplate of T5 and inferior endplate of T12; and 7) sagittal vertical axis (SVA), the horizontal distance between $\mathrm{C} 7$ plumb-line and the posterosuperior corner of S1, positive if $\mathrm{C} 7$ plumb-line is behind the posterosuperior corner of S1, or otherwise negative (Fig. 2). All values were measured three times and the average numbers were acquired.

All subjects were categorized by Roussouly classification according to their pelvic and lumbar alignments (Fig. 1). We compared the data of groups and radiologic parameters, according to Roussouly's classification, with those of other countries.

\section{Statistical analysis}

Statistical analyses were conducted with SPSS ver. 12.0 (SPSS Inc., Chicago, IL, USA). Descriptive statistics were provided in the form of mean \pm standard deviation and the confidence interval was set at 95\%. Lilliefors test was used to examine whether the parameters were normally distributed. Two 
tailed unpaired student t-test were utilized to compare between different groups. Statistical significance was set at 0.05 .

\section{RESULTS}

There was no significant difference in average age between men and women $(p=0.887)$. No statistical differences was noted in LL, TK, and SS ( $p>0.05)$ (Table 1). There were significant differences in the values of PI, PT, SSA, and SVA between two genders $(p<0.05)$. Those difference was attributed to relative pelvic retroversion due to female reproductive system.

In this study, 125 subjects among 252 (49.6\%) belonged to Roussouly type 3 (namely neutral) (Table 2). There were also 58 (23\%), 33 (13.1\%), and 36 (14.3) subjects in type 1, 2, and 4 (namely non-neutral), respectively. In a male group, there were type $1(36,23.8 \%)$, type $2(26,17.2 \%)$, type $3(70,46.4 \%)$, and type $4(19,12.6 \%)$. Type 1 (22, 21.8\%), type $2(13,12.9 \%)$, type $3(49,48.5 \%)$, and type $4(16,15.8 \%)$ were noted in a female group. Type 3 subjects were $46.4 \%$ of males and $55.4 \%$ of females (Table 2). No difference was revealed between males and females in the distribution of Roussouly types, or in comparison between the neutral group (type 3 ) and non-neutral (type 1, 2, and 4) $(p=0.275)$ (Table 2).

All sagittal radiological parameters were significantly different among four Roussouly types except sacral slope (Tables 3 and 4). Lumbar lordosis and Pelvic incidence was increased from type 1 to $4\left(\mathrm{LL}: 47.8 \pm 9.3^{\circ}, 37.8 \pm 5.5^{\circ}, 53.5 \pm 6.7^{\circ}, 67.5 \pm 5.6^{\circ}\right.$; PI : $\left.38.3 \pm 2.6^{\circ}, 46.1 \pm 8.6^{\circ}, 47.6 \pm 6.4^{\circ}, 60.4 \pm 6.8^{\circ}\right)$ Sagittal vertical axis were different among types $(28.71 \pm 28.12,12.15 \pm 32.4$, $15.44 \pm 36.14,1.05 \pm 8.99$ ). Type 1 had the largest one and type 4 the smallest. There were also no statistical significance in spinosacral angle except between type 1, 2, 3, and 4 groups $(p<0.05)$ (Tables 3 and 4).

\section{DISCUSSION}

The balance of the sagittal plane of the spine is essential for function as well as body shape, and the relation of the spine to pelvis is becoming increasingly important ${ }^{3)}$. The spinopelvic alignment play an important role in maintaining an effective posture in normal and spinal diseases ${ }^{14)}$. Sagittal alignment of the human spine and pelvis in standing and walking position is highly various in different individuals ${ }^{9)}$. In simple overviews, the human spine has kyphosis in thoracic spine curve and lordosis in cervical and lumbar ones ${ }^{12)}$. In historical review, Stagnara et al. ${ }^{13)}$ classified the vertebral curves into normal, kyphotic, and lordotic according to their shape and degree, and Dubousset regarded the pelvis as the supporting segment between the vertebrae and the lower extremities. Evcik and Yucel $^{2}$ claimed that there were statistical difference in thoracolumbar sagittal curve between patients who had chronic low back pain and controls who had acute back pain. The decrease of lumbar lordosis with an anterior shift of the sagittal vertical axis generates spinal symptoms and signs with spinal misalignment which is difficult to treat ${ }^{10)}$. So, many authors have commented on the importance of preserving lumbar lordosis during lumbar spine fusion and avoidance of postoperative flat back ${ }^{6)}$.

Not only regional spinal sagittal balance but also global spinal sagittal balance is crucial in every aspect of spinal treatment ${ }^{1}$. First of all, global spinal sagittal balance pattern would be the base of on-going studies in Korean. This prospective study focused on the groupings of the global sagittal postures of asymptomatic Korean adults in order to provide some references for future studies. For a comparison between the Korean and other ethnicities, we intentionally adopted a closely similar radiographic protocols as matched to those of Roussouly's. The difference of imaging methods between Roussouly's study and this study was both arm and shoulder position.

Table 2. Distribution of Roussouly classification

\begin{tabular}{|c|c|c|c|c|c|}
\hline & All $(n=252)$ & Male $(n=151)$ & Female $(n=101)$ & $p$-value* & $p$-value \\
\hline Type 1 & $58(23.0)$ & $36(23.8)$ & $22(21.8)$ & 0.452 & \\
\hline Type 2 & 33 (13.1) & $26(17.2)$ & $13(12.9)$ & 0.102 & \\
\hline Type 3 & 125 (49.6) & $70(46.4)$ & $49(48.5)$ & 0.342 & 0.275 \\
\hline Type 4 & $36(14.3)$ & 19 (12.6) & $16(15.8)$ & 0.215 & \\
\hline
\end{tabular}

Values are presented as number (\%). ${ }^{*} p$-value for the comparison distribution of Roussouly classification between male and female, ${ }^{\dagger} p$-value for the comparison between neutral Roussouly type (type 3) and non-neutral ones (type 1, 2, and 4) 
Table 3. Sagittal parameters according to types of Roussouly classification

\begin{tabular}{lcccc}
\hline Parameter & Type 1 & Type 2 & Type 3 & Type 4 \\
\hline Thoracic kyphosis $\left(^{\circ}\right)$ & $23.1 \pm 4.4$ & $17.4 \pm 8.7$ & $27.8 \pm 9.9$ & $24.6 \pm 7.4$ \\
Lumbar lordosis $\left(^{\circ}\right)$ & $47.8 \pm 9.3$ & $37.8 \pm 5.5$ & $53.5 \pm 6.7$ & $67.5 \pm 5.6$ \\
Sacral slope $\left(^{\circ}\right)$ & $22.8 \pm 3.1$ & $30.4 \pm 2.4$ & $38.9 \pm 2.8$ & $53.7 \pm 5.1$ \\
Pelvic incidence $\left(^{\circ}\right)$ & $38.3 \pm 2.6$ & $46.1 \pm 8.6$ & $47.6 \pm 6.4$ & $60.4 \pm 6.8$ \\
Pelvic tilt $\left({ }^{\circ}\right)$ & $17.6 \pm 3.9$ & $18.6 \pm 6.7$ & $13.8 \pm 2.1$ & $17.08 \pm 7.7$ \\
Spinosacral angle $\left(^{\circ}\right)$ & $133.3 \pm 9.8$ & $125.8 \pm 3.9$ & $132.6 \pm 4.8$ & $142.4 \pm 4.8$ \\
Sagittal vertical axis $(\mathrm{mm})$ & $28.71 \pm 28.1$ & $12.15 \pm 32.4$ & $15.44 \pm 36.1$ & $1.05 \pm 9.0$ \\
\hline
\end{tabular}

Values are presented as mean \pm standard deviation

Table 4. $p$-values for the comparison of any two Roussouly types

\begin{tabular}{|c|c|c|c|c|c|c|}
\hline Parameter & Type 3 vs. type 1 & Type 3 vs. type 2 & Type 3 vs. type 4 & Type 1 vs. type 2 & Type 1 vs. type 4 & Type 2 vs. type 4 \\
\hline Thoracic kyphosis $\left({ }^{\circ}\right)$ & 0.356 & 0.000 & 0.750 & 0.001 & 0.147 & 0.000 \\
\hline Lumbar lordosis $\left({ }^{\circ}\right)$ & 0.002 & 0.001 & 0.001 & 0.001 & 0.002 & 0.001 \\
\hline Pelvic incidence $\left(^{\circ}\right)$ & 0.000 & 0.203 & 0.000 & 0.055 & 0.005 & 0.000 \\
\hline Pelvic tilt $\left(^{\circ}\right)$ & 0.951 & 0.342 & 0.005 & 0.498 & 0.003 & 0.002 \\
\hline Spinosacral angle $\left({ }^{\circ}\right)$ & 0.000 & 0.261 & 0.000 & 0.586 & 0.000 & 0.000 \\
\hline Sagittal vertical axis (mm) & 0.269 & 0.458 & 0.875 & 0.459 & 0.006 & 0.478 \\
\hline
\end{tabular}

Statistically significant at $p<0.05$

In this study, fists-on-clavicle position was performed for the avoidance of covering spinal column by arms and shoulders. To show the difference in the distribution of the sagittal postural patterns between males and females, this study separated the subjects according to gender.

252 asymptomatic adult were enrolled, whose average PI value was $45.1 \pm 7.5^{\circ}$ (Table 1), lower than that of Caucasian $\left(51.7 \pm 55.0^{\circ}\right)$, similar to Chinese's one $\left(43.3 \pm 5.1^{\circ}\right)^{12,14)}$. PI value has been known to constant value, a fixed anatomic relationship between the sacrum and the pelvis, irrespective of aging, which is pivotal value of spinal correction surgery ${ }^{16)}$. This result might be related to the smaller average body size of Korean population than that of the Caucasian adults ${ }^{8,18)}$. Our study found that regardless of spinal symptom, 49.6\% of Korean adults possessed a neutral sagittal posture, namely Roussouly type 3 , which was higher than $37.5 \%$ of Caucasian adults, as previous study, and which was no difference with Chinese, $47.8 \%$ as by $\mathrm{Hu}$ et al. ${ }^{5)}$ and Roussouly et al. ${ }^{12)}$. As we used similar imaging protocol employed by Roussouly et al. ${ }^{12)}$, this difference between the two ethnics could not be attributed to imaging methods and measurements.
In this study, there were no differences of TK, LL, SS, PI, PT, SVA, and SSA values in gender as same as Caucasian study. But, in Chinese study in Asian ethnicity, females had higher SVA and SSA values than males and this was reflected by a higher proportion of Roussouly type 3 and type 4's in female group than male. Average PT was $13.8 \pm 2.1^{\circ}$, type 4 had the smallest value than others. It means that the anterior flexion of pelvis to spine have lower PT for the compensation of posture because the value of PT and SS changes according to the position and posture of the spine and pelvis.

All the sagittal parameters except SS were significantly different between Roussouly types (Tables 3 and 4), and this is a reflection of the usefulness of Roussouly classification on sagittal postural alignments.

In general, Correlation between spinal parameter and pelvic parameter is important for spinal sagittal balance ${ }^{11)}$. The most important correlation is between the PI as anatomical index and the SS as the positional index ${ }^{7,15)}$. The second most important correlation is between the SS and the LL, and the LL and the TK have little correlation ${ }^{7,8)}$. The PI is the sum of the SS and the PT. There is no change in the PI with the rotation of 
the pelvis. However, when the SS increases, the PT decreases, and when the SS increases, the PT increases ${ }^{3,4}$. As a whole, all radiologic parameter values of this study follow above the hypothesis. From type 1 to 4, the LL increase, more lordosis but no change occur at the TK. Regardless of type, the larger the SS, the smaller PT becomes a constant the PI. According to general spinal sagittal pattern, Lumbar parameters and pelvic parameters are changed for compensatory mechanism ${ }^{15}$. The decrease of lumbar lordosis and increase of thoracic kyphosis due to spinal degeneration is the main cause of sagittal imbalance. In type 1, 2, LL is lower than that of type 3, the PT is higher enough to compensate for lower lordosis. This is due to reduced LL followed by the facet degeneration, collapsed disc etc. In order to optimize the treatment of spinal sagittal imbalance and to avoid overlooking the severity of degenerative diseases, it is important to identify and understand the different patterns of these global spinal sagittal balance pattern. The sagittal balance is related to the pelvis and hip in addition to the spine, and the pelvic and spinal parameters show a close correlation with each other. Therefore, it is essential to measure the pelvic parameters as well as the spinal parameters. For this purpose, we should understand global sagittal balance of normal state as well as diseased state.

There were some limitations in our study. First, the recruitment bias seemed to be inevitable, enrolled subjects had not been evenly distributed for the representation of normal Korean adult. Second, radiologic imaging method was not same, so direct comparison among other ethnic group was not maintained. Third, more intense intraobserver and interobserver reliability was needed for the evaluation of measurement of parameters. Finally, as this was preliminary screening study, more careful designed future study for sagittal postural alignment are needed for clinical interpretations.

\section{CONCLUSION}

Registering 252 asymptomatic Korean adults, this prospective study found that $49.6 \%$ of asymptomatic Korean adults possessed a sagittal posture of Roussouly type 3. All radiologic parameters follows general concept of spinal sagittal balance pattern. Overall, this study might be a basis for further investigation of spinal sagittal balance.

\section{- Acknowledgements}

The study protocol was approved by the institutional review board of Ewha Womans Mokdong Hospital (IRB No. 2016-08-023). Informed consent was confirmed by the IRB.

\section{References}

1. Diebo BG, Henry J, Lafage V, Berjano $P$ : Sagittal deformities of the spine: factors influencing the outcomes and complications. Eur Spine $J$ 24 Suppl 1 : S3-S15, 2015

2. Evcik $D$, Yucel $A$ : Lumbar lordosis in acute and chronic low back pain patients. Rheumatol Int 23 : 163-165, 2003

3. Garbossa D, Pejrona M, Damilano M, Sansone V, Ducati A, Berjano P : Pelvic parameters and global spine balance for spine degenerative disease: the importance of containing for the well being of content. Eur Spine J 23 Suppl 6 : 616-627, 2014

4. Hasegawa K, Okamoto M, Hatsushikano S, Shimoda H, Ono M, Watanabe $K$ : Normative values of spino-pelvic sagittal alignment, balance, age, and health-related quality of life in a cohort of healthy adult subjects. Eur Spine J 25 : 3675-3686, 2016

5. Hu P, Yu M, Sun Z, Li W, Jiang L, Wei F, et al. : Analysis of global sagittal postural patterns in asymptomatic Chinese adults. Asian Spine J 10 : 282-288, 2016

6. Kostuik JP, Maurais GR, Richardson WJ, Okajima Y : Combined single stage anterior and posterior osteotomy for correction of iatrogenic lumbar kyphosis. Spine (Phila Pa 1976) 13 : 257-266, 1988

7. Labelle H, Mac-Thiong JM, Roussouly P : Spino-pelvic sagittal balance of spondylolisthesis: a review and classification. Eur Spine J 20 Suppl 5 : 641-646, 2011

8. Lee JH, Kim KT, Suk KS, Lee SH, Jeong BO, Kim JS, et al. : Analysis of spinopelvic parameters in lumbar degenerative kyphosis: correlation with spinal stenosis and spondylolisthesis. Spine (Phila Pa 1976) 35 : E1386-E1391, 2010

9. Mac-Thiong JM, Roussouly P, Berthonnaud E, Guigui P : Sagittal parameters of global spinal balance: normative values from a prospective cohort of seven hundred nine Caucasian asymptomatic adults. Spine (Phila Pa 1976) 35 : E1193-E1198, 2010

10. Noun Z, Lapresle P, Missenard G : Posterior lumbar osteotomy for flat back in adults. J Spinal Disord 14 : 311-316, 2001

11. Rousseau MA, Lazennec JY, Tassin JL, Fort D; Groupe d'Etude la Scoliose [French Scoliosis Study Group] : Sagittal rebalancing of the pelvis and the thoracic spine after pedicle subtraction osteotomy at the lumbar level. J Spinal Disord Tech 27 : 166-173, 2014

12. Roussouly P, Gollogly S, Berthonnaud E, Dimnet J : Classification of the normal variation in the sagittal alignment of the human lumbar spine and pelvis in the standing position. Spine (Phila Pa 1976) 30 : 346 353, 2005

13. Stagnara P, De Mauroy JC, Dran G, Gonon GP, Costanzo G, Dimnet J, 
et al. : Reciprocal angulation of vertebral bodies in a sagittal plane: approach to references for the evaluation of kyphosis and lordosis. Spine (Phila Pa 1976) 7 : 335-342, 1982

14. Vaz G, Roussouly P, Berthonnaud E, Dimnet J : Sagittal morphology and equilibrium of pelvis and spine. Eur Spine J 11 : 80-87, 2002

15. Vrtovec $T$, Janssen MM, Likar $B$, Castelein RM, Viergever MA, Pernuš $\mathrm{F}: \mathrm{A}$ review of methods for evaluating the quantitative parameters of sagittal pelvic alignment. Spine J 12 : 433-446, 2012

16. Yagi M, Takeda K, Machida M, Asazuma T : Discordance of gravity line and C7PL in patient with adult spinal deformity--factors affecting the occiput-trunk sagittal discordance. Spine J 15 : 213-221, 2015

17. Yu M, Silvestre C, Mouton T, Rachkidi R, Zeng L, Roussouly P : Analysis of the cervical spine sagittal alignment in young idiopathic scoliosis: a morphological classification of 120 cases. Eur Spine J 22 : 2372-2381, 2013

18. Zhu Z, Xu L, Zhu F, Jiang L, Wang Z, Liu Z, et al. : Sagittal alignment of spine and pelvis in asymptomatic adults: norms in Chinese populations. Spine (Phila Pa 1976) 39 : E1-E6, 2014 